\title{
Performance Scaling in Magnetized Liner Inertial Fusion Experiments
}

\author{
M. R. Gomez®, ${ }^{1, *}$ S. A. Slutz, ${ }^{1}$ C. A. Jennings, ${ }^{1}$ D. J. Ampleford $\odot,{ }^{1}$ M. R. Weis $\odot,{ }^{1}$ \\ C. E. Myers $\odot,{ }^{1}$ D. A. Yager-Elorriaga $\odot,{ }^{1}$ K. D. Hahn $\odot,{ }^{2}$ S. B. Hansen, ${ }^{1}$ E. C. Harding, ${ }^{1}$ A. J. Harvey-Thompson, ${ }^{1}$ \\ D. C. Lamppa, ${ }^{1}$ M. Mangan, ${ }^{1}$ P. F. Knapp, ${ }^{1}$ T. J. Awe, ${ }^{1}$ G. A. Chandler, ${ }^{1}$ G. W. Cooper, ${ }^{1,3}$ J. R. Fein $\odot,{ }^{1}$ M. Geissel $\odot,{ }^{1}{ }^{1}$ M. \\ E. Glinsky $\odot,{ }^{1}$ W. E. Lewis, ${ }^{1}$ C. L. Ruiz $\odot,{ }^{1}$ D. E. Ruiz $\odot,{ }^{1}$ M. E. Savage, ${ }^{1}$ P. F. Schmit $\odot,{ }^{1}$ I. C. Smith, ${ }^{1}$ J. D. Styron, ${ }^{3}$ \\ J. L. Porter, ${ }^{1}$ B. Jones, ${ }^{1}$ T. R. Mattsson $\odot,{ }^{1}$ K. J. Peterson $\odot,{ }^{1}$ G. A. Rochau, ${ }^{1}$ and D. B. Sinars $\odot^{1}$ \\ ${ }^{1}$ Sandia National Laboratories, Albuquerque, New Mexico 87185, USA \\ ${ }^{2}$ Lawrence Livermore National Laboratory, Livermore, California 94550, USA \\ ${ }^{3}$ University of New Mexico, Albuquerque, New Mexico 87131, USA
}

(Received 17 January 2020; revised 31 July 2020; accepted 27 August 2020; published 9 October 2020)

\begin{abstract}
We present experimental results from the first systematic study of performance scaling with drive parameters for a magnetoinertial fusion concept. In magnetized liner inertial fusion experiments, the burnaveraged ion temperature doubles to $3.1 \mathrm{keV}$ and the primary deuterium-deuterium neutron yield increases by more than an order of magnitude to $1.1 \times 10^{13}$ ( $2 \mathrm{~kJ}$ deuterium-tritium equivalent) through a simultaneous increase in the applied magnetic field (from 10.4 to $15.9 \mathrm{~T}$ ), laser preheat energy (from 0.46 to $1.2 \mathrm{~kJ}$ ), and current coupling (from 16 to $20 \mathrm{MA}$ ). Individual parametric scans of the initial magnetic field and laser preheat energy show the expected trends, demonstrating the importance of magnetic insulation and the impact of the Nernst effect for this concept. A drive-current scan shows that present experiments operate close to the point where implosion stability is a limiting factor in performance, demonstrating the need to raise fuel pressure as drive current is increased. Simulations that capture these experimental trends indicate that another order of magnitude increase in yield on the $Z$ facility is possible with additional increases of input parameters.
\end{abstract}

DOI: 10.1103/PhysRevLett.125.155002

Achieving controlled thermonuclear fusion in the laboratory remains a scientific grand challenge with important applications in stockpile stewardship and the potential for future use in energy production. Despite this, it has proven difficult to confine a plasma with high enough density and sufficient temperature for the necessary duration to produce an output energy that exceeds the input [1]. An interesting path to ignition and high gain [2,3] is magnetoinertial fusion (MIF) [4-6], which bridges the gap between the long duration $\left(>10^{2} \mathrm{~s}\right)$, low density $\left(10^{20} \mathrm{~m}^{-3}\right)$ magnetic confinement schemes [7,8] and the short duration $\left(<10^{-10} \mathrm{~s}\right)$, high density $\left(10^{32} \mathrm{~m}^{-3}\right)$ inertial confinement schemes [9-11].

Magnetized liner inertial fusion (MagLIF) [12] is a MIF concept that utilizes a kilojoule-class laser $[13,14]$ to raise the fuel adiabat and an applied axial magnetic field [15] to limit conduction losses throughout the magnetically driven implosion [16]. MagLIF has shown considerable promise [17] on the $Z$ facility [18,19] by demonstrating

Published by the American Physical Society under the terms of the Creative Commons Attribution 4.0 International license. Further distribution of this work must maintain attribution to the author(s) and the published article's title, journal citation, and DOI. thermonuclear neutron generation at fusion-relevant temperatures and densities [17,20,21] with sufficient fuel magnetization at stagnation to trap fusion alpha particles [22-24]. MagLIF has also been demonstrated to work at $1 / 1000$ energy scale and $1 / 10$ spatial scale (relative to $Z$ experiments) on the OMEGA laser facility $[25,26]$.

This Letter describes the first successful experimental demonstration of performance scaling in a MIF concept; significantly increased neutron production was observed with greater applied magnetic field, laser preheat energy, and drive current. The maximum performance in MagLIF now exceeds $10^{13}$ primary deuterium-deuterium (DD) neutrons ( $2 \mathrm{~kJ}$ deuterium-tritium equivalent), representing a factor of 3 increase over the highest previously reported MagLIF experimental yield [27]. Trends observed in parametric scans of individual input parameters, as well as the improved performance observed when all inputs were simultaneously increased, are consistent with expectations based on 2D magnetohydrodynamics calculations [28] performed in LASNEX. The LASNEX model predicts that further increases in preheat energy, magnetic field, and current could enable another order of magnitude increase in yield on the $Z$ facility [28], as well as multimegajoule yields on a future pulsed power driver [2,3]. 
The recent significant increase in performance demonstrated in MagLIF experiments leveraged three key improvements to the platform. First, the target was redesigned to eliminate nonberyllium fuel-facing components, which were a significant source of enhanced radiative loss in previous experiments [29], especially as preheat energy was increased [30]. Second, an improved preheat configuration was developed, which reduced laser plasma instabilities as well as the injection of laserentrance-hole window material into the fuel [31]. This configuration utilizes a distributed phase plate to create a more uniform intensity laser spot and a laser pulse shape with a low energy $(0.02 \mathrm{~kJ})$ prepulse, $0.3 \mathrm{~kJ}$ foot, and $2.2 \mathrm{~kJ}$ main pulse to deposit up to $1.4 \mathrm{~kJ}$ in the fuel. Note that all subsequent preheat energy values reported in this Letter refer to the energy deposited in the fuel (determined using the measured energy at the output of the laser and deposition fractions based on off-line experiments using similar target configurations and laser pulses [27,30]). Finally, an advanced magnetic field coil [32] and more efficient final transmission line were developed [Fig. 1(c)], enabling simultaneous increases in the applied magnetic field (up to $16 \mathrm{~T}$ average) and peak current (up to $20 \mathrm{MA}$ ). The increase in peak current is primarily due to a reduction of current losses in the convolute [33] and final transmission line, which impact the shape of the load current as well [Fig. 1(d)].

The highest-performing experiment was conducted with $15.9 \mathrm{~T}, 1.2 \mathrm{~kJ}$ preheat energy, and $20 \mathrm{MA}$ peak load current, producing $1.1 \mathrm{e} 13 \mathrm{DD}$ yield (measured via indium activation) with a $3.1 \mathrm{keV}$ burn-weighted average ion temperature (inferred via forward fit to neutron time-of-flight spectra $[30,35,36])$. The neutron yield was isotropic, and the primary neutron spectrum was Gaussian, which is consistent with previous observations that these sources are thermonuclear $[17,20]$. Additionally, this experiment is part of a larger dataset in which the neutron yield increases as expected with ion temperature for a thermonuclear source (Fig. 2). Also noted in Fig. 2 is an experiment with similar initial fuel density, preheat energy, and applied magnetic field to the earliest MagLIF experiments [17], which shows the gains realized through the improvements to the MagLIF platform. To better understand how the increase in each input parameter contributed to the improved performance in this experiment, a series of individual parametric scans of preheat energy, magnetic field, and peak load current were conducted.

Using the target and load hardware geometries shown in Figs. 1(a) and 1(b), respectively, a parametric scan of preheat energy was performed. The initial fuel density, applied magnetic field, and peak current drive remained fixed at $0.68 \mathrm{mg} / \mathrm{cm}^{3}, 10 \mathrm{~T}$, and $16 \mathrm{MA}$, respectively, and the preheat energy was varied between 0 and $1.4 \mathrm{~kJ}$. A significant increase in the primary DD neutron yield with preheat energy was observed up to about $0.8 \mathrm{~kJ}$; then yield remained flat up to $1.4 \mathrm{~kJ}$ [Fig. 3(a)]. The burn-averaged ion temperature [Fig. 3(b)] follows a similar trend, increasing up to a preheat energy of $0.8 \mathrm{~kJ}$, then remaining flat with increased preheat.

The observed trend is consistent with expectations [12,28] when accounting for the Nernst effect. In MagLIF, the preheat laser interacts with a fraction of the fuel volume, creating a higher-temperature region on the axis surrounded by a cooler layer of fuel [37]. The Nernst effect enhances the transport of magnetic flux away from hot regions surrounded by steep temperature

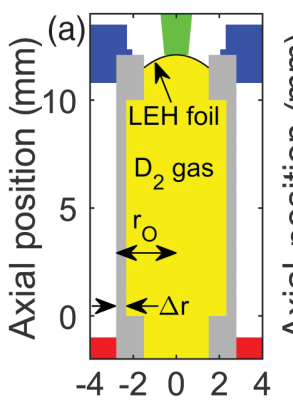

Radial position (mm)

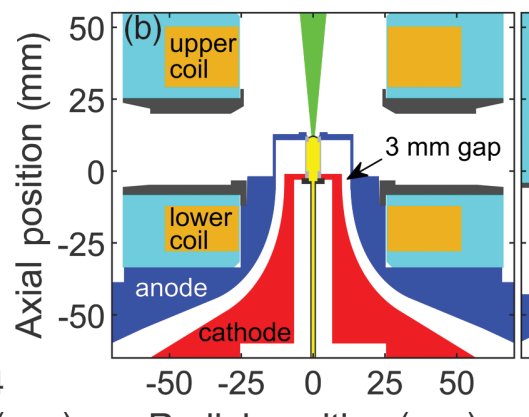

Radial position $(\mathrm{mm})$
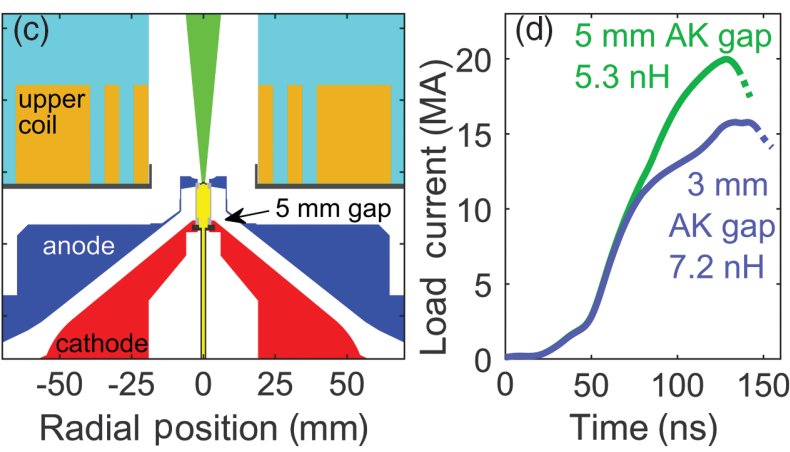

FIG. 1. (a) Cross section of the target configuration used in these experiments. The laser enters the target through the laser-entrancehole (LEH) window at $z=12.6 \mathrm{~mm}$. The imploding portion of the target spans from $z=0$ to $10 \mathrm{~mm}$. The target aspect ratio is defined as the outer radius divided by the wall thickness ( $\left.\mathrm{AR}=r_{O} / \Delta r\right)$. (b),(c) Cross sections of the load configurations used in the experiments conducted at 16 and $20 \mathrm{MA}$, respectively. The target is shown in gray, fuel in yellow, anode in blue, cathode in red, coil windings in orange, internal coil support in cyan, external coil support in dark gray, and laser in green. With a single coil above the target, the amplitude of the axial field in (c) decreased from the top to the bottom of the target by $30 \%$. The average field is reported for experiments using this configuration. (d) Plot of the load current (determined with velocimetry [34]) for the two transmission line geometries. The transition to dashed lines after peak current indicates reduced fidelity of the velocimetry measurement due to relaxation of the drive pressure. The higher inductance and smaller anode-cathode gap geometry (b) has greater current loss, which impacts the shape and peak of the current pulse. 


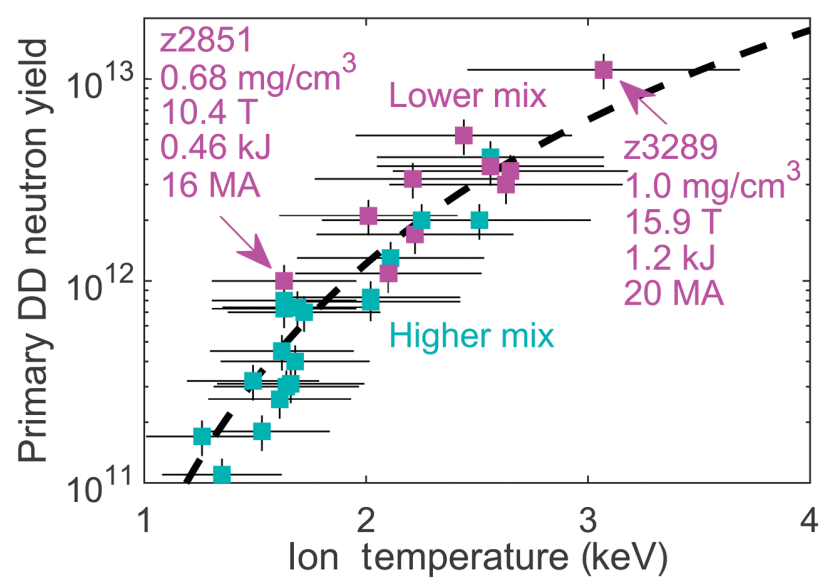

FIG. 2. Plot of the primary neutron yield as a function of ion temperature for all $10 \mathrm{~mm}$ tall, $5.58 \mathrm{~mm}$ outer diameter, $\mathrm{AR}=6$ MagLIF experiments (squares) with the reactivity parameter for DD- ${ }^{3} \mathrm{He}$ fusion [37] scaled by $3.9 \times 10^{38}$ overlaid (dashed line) for comparison. Experiments that utilized protocols to reduce radiative loss (magenta) tended to have higher ion temperatures and consequently neutron yields than those that did not (teal). Increasing from 0.68 to $1.0 \mathrm{mg} / \mathrm{cm}^{3}, 10.4$ to $15.9 \mathrm{~T}, 0.46$ to $1.2 \mathrm{~kJ}$, and 16 to $20 \mathrm{MA}$ doubled the ion temperature and increased the yield by an order of magnitude.

gradients [38,39]. Assuming a fixed fuel density and preheat volume, increasing the preheat energy produces higher on-axis temperatures and larger radial temperature gradients. This increases both the radial heat flux and magnetic flux losses from the hottest region of the fuel. Because the radial heat flux is inhibited by the axial magnetic field, the loss of magnetic flux in these regions further increases heat flux. The positive feedback between radial heat flux from the hot fuel on axis and the enhanced magnetic flux loss due to the Nernst effect prevents continued increases in fuel temperature with increasing preheat energy unless the applied magnetic field is also increased [28].

High-energy (11-17 MeV) neutrons created through fusion reactions between DD fusion tritons and the deuterium fuel can be used to assess the magnetization of the fuel column at stagnation [22,23]. Using the ratio of the primary neutron yield to secondary neutron yield (measured via copper activation) and the secondary neutron spectra (obtained via neutron time of flight), the inferred magnetic field-radius product was observed to decrease with increasing preheat energy [Fig. 3(c)], while the measured convergence ratio (CR) (based on x-ray selfemission imaging) remained approximately constant. This decrease in magnetization with increasing preheat energy deposition is consistent with expectations when accounting for the Nernst effect. In future experiments with deuteriumtritium fuel, retention of magnetic flux in the fuel will be critical to trap fusion alpha particles [12]. This provides motivation for operating with a preheat energy at the low
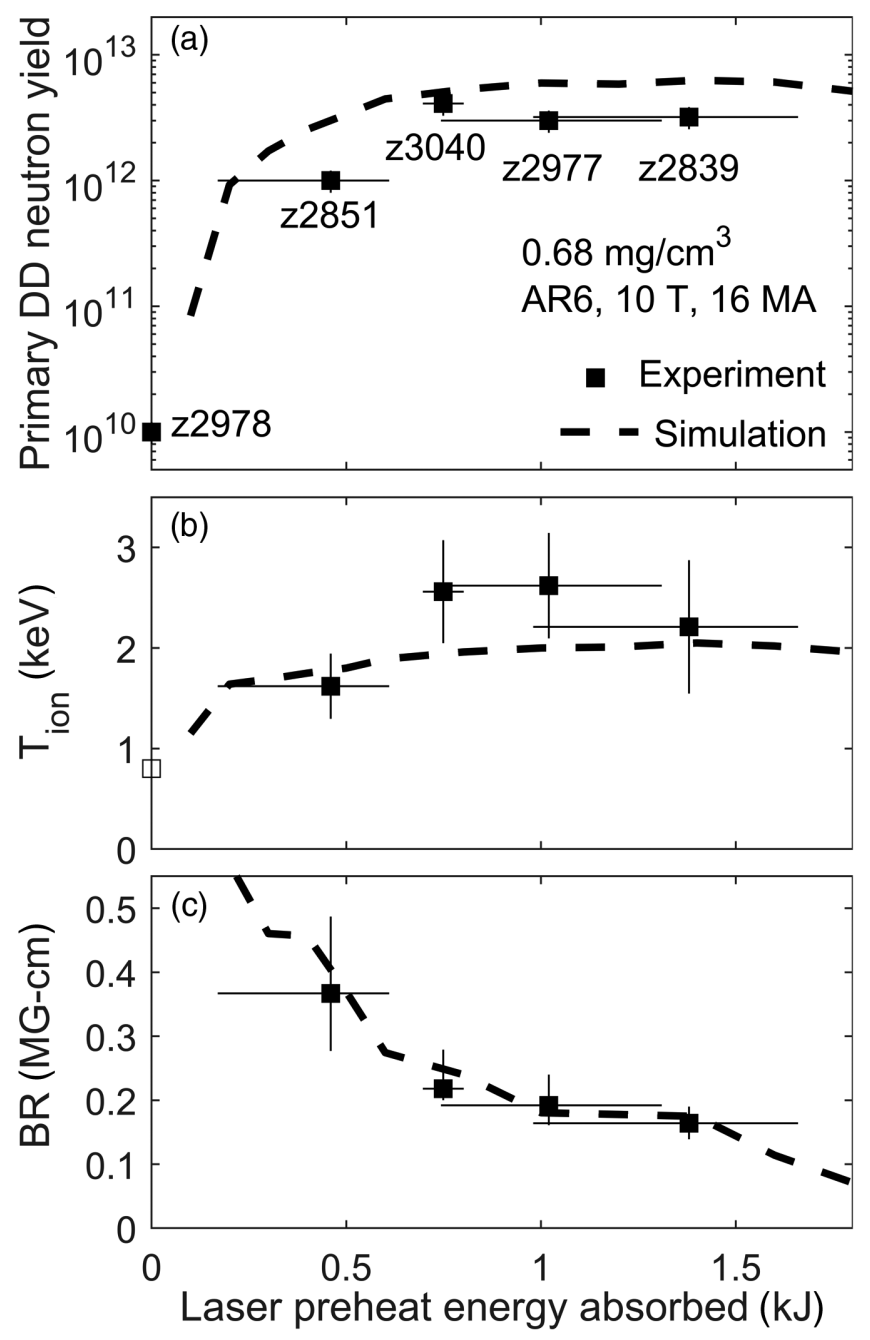

FIG. 3. (a),(b) Plots of primary neutron yield and burn-averaged ion temperature as a function of laser preheat energy absorbed in the fuel, respectively, showing increasing performance followed by a plateau. (c) Plot of the magnetic field-radius product (BR) as a function of preheat energy showing a decay in magnetization at stagnation as preheat energy increases, which is consistent with increasing magnetic flux loss due to the Nernst effect. Experimental values (solid squares) and simulation results (dashed lines) are shown. An experiment with zero preheat energy produced $10^{10}$ primary neutrons (about 3 times the detection threshold) but provided an insufficient signal to determine the ion temperature or BR. The ion temperature for this experiment (shown as an open square) was estimated using the reactivity curve in Fig. 2 and the measured yield.

end of the ion temperature-yield plateau, where magnetization is maximized while maintaining peak stagnation performance.

To test the impact of increasing initial magnetization on target performance, a series of experiments were conducted with the transmission line geometry in Fig. 1(b). For this parametric scan of the applied magnetic field, the targets had a wall thickness of $242 \mu \mathrm{m}$ (reduced from $465 \mu \mathrm{m}$ ) and a $75 \mu \mathrm{m}$ dielectric coating was applied to the exterior of the 


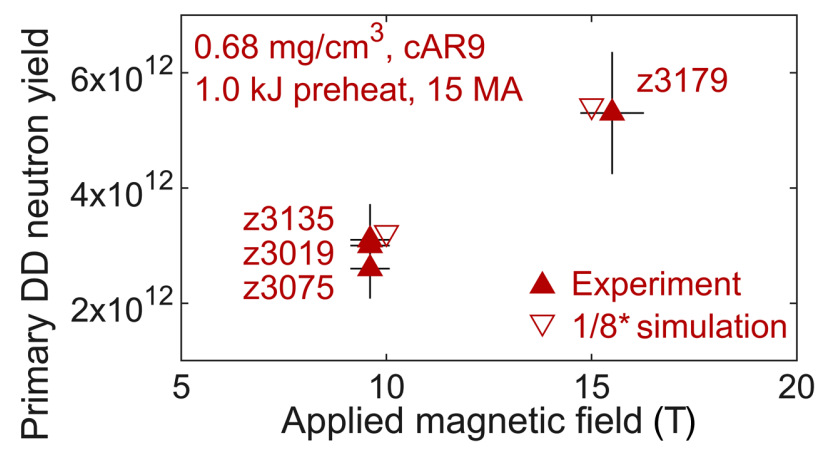

FIG. 4. Plot of primary neutron yield as a function of applied magnetic field. Experiments with dielectric-coated targets that have been mass matched to $\mathrm{AR}=9$ targets (cAR9) [40] are shown as solid triangles, and 2D LASNEX simulations with AR9 targets (scaled by $1 / 8$ ) are plotted as inverted open triangles. The CR in these simulations was between 46 and 49, resulting in a simulation-to-experimental-yield ratio of approximately 8 .

target to stabilize the implosion [40]. The peak load current was $15 \mathrm{MA}$, the fuel density was $0.68 \mathrm{mg} / \mathrm{cm}^{3}$, and the preheat energy was approximately $1.0 \mathrm{~kJ}$. The applied magnetic field was increased from 9.7 to $15.8 \mathrm{~T}$, which resulted in an $80 \%$ increase in primary neutron yield (Fig. 4) and a 30\% increase in burn-averaged ion temperature, which is consistent with enhanced thermal insulation provided by the higher magnetic field.

In contrast to the preheat energy and applied magnetic field scans, holding the other input parameters fixed while increasing the peak load current from 16 to 20 MA showed no significant change in primary neutron yield or burnaveraged ion temperature. In these experiments the initial fuel density was $0.68 \mathrm{mg} / \mathrm{cm}^{3}$, the applied magnetic field was approximately $10 \mathrm{~T}$, and the preheat energy was approximately $1.0 \mathrm{~kJ}$. Note that different final transmission line geometries were used for the low-current [Fig. 1(b)] and high-current [Fig. 1(c)] cases, which resulted in different current pulse shapes between the two cases [Fig. 1(d)], and the higher-current case also had a less axially uniform magnetic field (the impact of this difference is expected to be minimal based on simulations).

Previous experience with other $Z$-pinch fusion concepts [41,42] as well as analytic arguments [43] suggests that the yield should scale roughly as peak current to the fourth power, which initially appears to be inconsistent with these results. However, without any other changes, increasing peak current should also produce a higher CR, and the observed CR did not significantly change between the high- and low-current cases (Fig. 5). Interestingly, there are significantly larger variations in the radial extent for the higher-current experiment, which is consistent with increased liner instability development. This suggests that instabilities can limit the potential gains provided by greater drive current. Notably, the highestperforming MagLIF experiment balanced the increase in

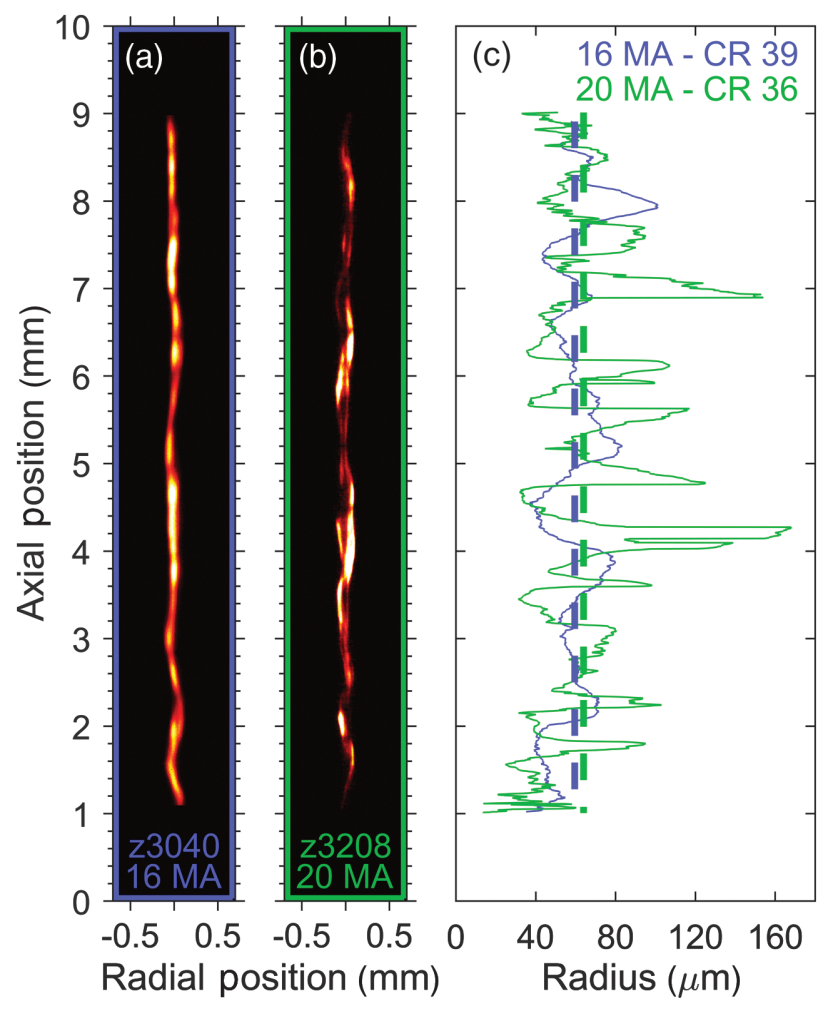

FIG. 5. (a),(b) X-ray self-emission images obtained with a spherical crystal imager in experiments with 16 and 20 MA peak load currents, respectively, while other parameters remained fixed. The transverse spatial resolution was approximately 40 and $20 \mu \mathrm{m}$ for (a) and (b), respectively, which accounts for some of the increased structure in (b). (c) Plot of the inferred radius [30] as a function of axial position (solid lines) and average radius (dashed lines) for the two experiments.

drive pressure supplied by the higher current with an increase in internal fuel pressure due to the higher preheat energy, applied magnetic field, and initial fuel density, resulting in a similar measured $\mathrm{CR}=35$. The observed increase in yield in this experiment was greater than expected when only accounting for the increase in preheat energy and applied magnetic field, indicating that increased current did have a positive impact when driving a target with sufficient fuel pressure.

Two-dimensional magnetohydrodynamics simulations conducted in LASNEX reproduce the relative trends observed in the experimental parametric scans of preheat energy and applied magnetic field. The model reproduces the plateaus in primary neutron yield and ion temperature as well as the decay in magnetization at stagnation with increasing preheat energy when the Nernst effect is included. The model also matches the experimentally observed increase in ion temperature and neutron yield with increasing magnetic field. However, the model expected more than a factor of 3 increase in neutron yield as the peak load current was increased from 16 to $20 \mathrm{MA}$, and no change was observed experimentally. Note that 
while there was no measurable change in the experimental $\mathrm{CR}$ with the increase in current, the simulated $\mathrm{CR}$ increased from less than 40 to nearly 50 .

This discrepancy likely arises from the inability of 2D simulations to account for the observed 3D instability structures, allowing the simulated stagnation column to reach CRs beyond those possible in experiments. This enables the stagnation pressure, and consequently the neutron yield, to exceed the values observed experimentally by a significant amount. In cases where the experimental and simulated convergence was similar $(C R \approx 35)$, the simulated-to-experimental-yield ratio was about 2 or 3 to 1 ; however, when the simulated convergence $(C R \approx 45-50)$ significantly exceeded the experimental convergence, the yield ratio increased to around 8 to 1 . This breakdown in surrogacy as the simulated convergence gets large is consistent with observations in other inertial fusion concepts $[44,45]$ and inspires efforts to reduce the CR in future designs. Note that the consistency between LASNEX simulations and the highest-performing MagLIF experiment provides evidence that simulation surrogacy can be maintained while scaling up drive current in MagLIF, as long as the increased magnetic pressure is balanced by an increased fuel pressure that limits convergence.

With continued platform development, applied magnetic fields up to $30 \mathrm{~T}$ and 4-6 kJ preheat energies appear feasible on the $Z$ machine. With these constraints, a series of LASNEX calculations were conducted at peak load currents of approximately 16, 19, and 22 MA to determine the configuration that maximizes neutron yield while limiting CR to below 40 (Table I). Assuming that the simulated-to-experimental-yield ratio remains approximately 2 or 3 to 1 due to this constraint on CR, these calculations indicate that another order of magnitude increase in neutron yield is plausible.

TABLE I. Optimized LASNEX solutions at three current levels where the magnetic field and preheat energy were constrained to a maximum of $30 \mathrm{~T}$ and $6 \mathrm{~kJ}$, respectively. Note that for configurations with lower preheat and magnetic field that have been tested experimentally, when simulated convergence was around 35, simulations overpredicted experimental DD yield by about a factor of 2 to 3 .

\begin{tabular}{lccc}
\hline \hline Peak current (MA) & 16.6 & 19.2 & 22.3 \\
\hline$\rho_{\text {fuel }}\left(\mathrm{mg} / \mathrm{cm}^{3}\right)$ & 0.8 & 1.1 & 1.4 \\
Magnetic field $(\mathrm{T})$ & 30 & 30 & 30 \\
Preheat $(\mathrm{kJ})$ & 3.2 & 4.4 & 6.0 \\
DD yield & $3.9 \times 10^{13}$ & $1.6 \times 10^{14}$ & $4.5 \times 10^{14}$ \\
CR & 34 & 34 & 36 \\
$T_{\text {ion }}(\mathrm{keV})$ & 4.0 & 4.9 & 5.5 \\
$\mathrm{P}(\mathrm{Gbar})$ & 1.0 & 1.8 & 2.8 \\
$\tau(\mathrm{ns})$ & 4.4 & 3.7 & 3.1 \\
BR $(\mathrm{MG} \mathrm{cm})$ & 0.50 & 0.55 & 0.64 \\
\hline \hline
\end{tabular}

In summary, simultaneously increasing the applied magnetic field, preheat energy, and peak load current has significantly improved target performance in MagLIF, producing a primary neutron yield approximately a factor of 3 greater than the previous highest reported yield. Parametric scans of individual input parameters revealed the following: (1) the Nernst effect plays an important role in MagLIF as the preheat energy increases, limiting the fuel temperature for a fixed magnetic field, (2) increasing the applied magnetic field enables higher temperatures and neutron yields through reduced thermal conduction losses, and (3) target performance improves with increasing current only when there is also sufficiently increased fuel pressure to maintain approximately constant CR. Two-dimensional simulations generally match the observed trends in target performance, though simulatedto-experimental-yield ratios increase as simulated CRs become too large, indicating more strongly perturbative effects from implosion instabilities. Continued platform development is expected to enable an additional factor of 10 increase in neutron yield.

The authors would like to thank the $Z$ machine, ZBL, and $\mathrm{ABZ}$ operations teams and the target fabrication team for their contributions to this work. The authors would also like to thank K. Chandler for the useful discussions on the accessibility of the manuscript as well as the referees for the excellent constructive criticism of the initial submission. This work was supported by Sandia National Laboratories, a multimission laboratory managed and operated by the National Technology and Engineering Solutions of Sandia, LLC, a wholly owned subsidiary of Honeywell International, Inc., for the U.S. Department of Energy's National Nuclear Security Administration under Contract No. DE-NA0003525. This Letter describes objective technical results and analysis. Any subjective views or opinions that might be expressed in the Letter do not necessarily represent the views of the U.S. Department of Energy or the U.S. Government. The work of M. R. G. was supported in part by the Presidential Early Career Award for Scientists and Engineers.

*Corresponding author. mrgomez@sandia.gov

[1] J. D. Lawson, Some criteria for a power producing thermonuclear reactor, Proc. Phys. Soc. London Sect. B 70, 6 (1957).

[2] S. A. Slutz and R. A. Vesey, High-Gain Magnetized Inertial Fusion, Phys. Rev. Lett. 108, 025003 (2012).

[3] S. A. Slutz, W. A. Stygar, M. R. Gomez, K. J. Peterson, A. B. Sefkow, D. B. Sinars, R. A. Vesey, E. M. Campbell, and R. Betti, Scaling magnetized liner inertial fusion on $\mathrm{Z}$ and future pulsed-power accelerators, Phys. Plasmas 23, 022702 (2016). 
[4] I. R. Lindemuth and R. C. Kirkpatrick, Parameter space for magnetized fuel targets in inertial confinement fusion, Nucl. Fusion 23, 263 (1983).

[5] I. R. Lindemuth and M. M. Widner, Magnetohydrodynamic behavior of thermonuclear fuel in a preconditioned electron beam imploded target, Phys. Fluids 24, 746 (1981).

[6] M. M. Basko, A. J. Kemp, and J. Meyer-ter-Vehn, Ignition conditions for magnetized target fusion in cylindrical geometry, Nucl. Fusion 40, 59 (2000).

[7] M. Shimada et al., Progress in the ITER physics basis, Nucl. Fusion 47, S1 (2007).

[8] R. C. Wolf, C. D. Beidler, A. Dinklage, P. Helander, H. P. Laqua, F. Schauer, T. S. Pedersen, and F. Warmer, Wendelstein 7-X program-demonstration of a stellarator option for fusion energy, IEEE Trans. Plasma Sci. 44, 1466 (2016).

[9] J. Lindl, Development of the indirect-drive approach to inertial confinement fusion and the target physics basis for ignition and gain, Phys. Plasmas 2, 3933 (1995).

[10] O. A. Hurricane et al., Fuel gain exceeding unity in an inertially confined fusion implosion, Nature (London) 506, 343 (2014).

[11] R.S. Craxton et al., Direct-drive inertial confinement fusion: A review, Phys. Plasmas 22, 110501 (2015).

[12] S. A. Slutz, M. C. Herrmann, R. A. Vesey, A. B. Sefkow, D. B. Sinars, D. C. Rovang, K. J. Peterson, and M. E. Cuneo, Pulsed-power-driven cylindrical liner implosions of laser preheated fuel magnetized with an axial field, Phys. Plasmas 17, 056303 (2010).

[13] P. K. Rambo, I. C. Smith, J. L. Porter, M. J. Hurst, C. S. Speas, R. G. Adams, A. J. Garcia, E. Dawson, B. D. Thurston, C. Wakefield, J. W. Kellogg, M. J. Slattery, H. C. Ives, R. S. Broyles, J. A. Caird, A. C. Erlandson, J. E. Murray, W. C. Behrendt, N. D. Neilsen, and J. M. Narduzzi, Z-Beamlet: a multikilojoule, terawatt-class laser system, J. Opt. A 44, 2421 (2005).

[14] P. Rambo, J. Schwarz, M. Schollmeier, M. Geissel, I. Smith, M. Kimmel, C. Speas, J. Shores, D. Armstrong, J. Bellum, E. Field, and D. Kletecka, and J. Porter, Sandia's Z-backlighter laser facility, Proc. SPIEInt. Soc. Opt. Eng. 10014, 100140Z (2016).

[15] D. C. Rovang et al., Pulsed-coil magnet systems for applying uniform 10-30 $\mathrm{T}$ fields to centimeter-scale targets on Sandia's Z facility, Rev. Sci. Instrum. 85, 124701 (2014).

[16] M.E. Cuneo et al., Magnetically driven implosions for inertial confinement fusion at sandia national laboratories, IEEE Trans. Plasma Sci. 40, 3222 (2012).

[17] M. R. Gomez et al., Experimental Demonstration of FusionRelevant Conditions in Magnetized Liner Inertial Fusion, Phys. Rev. Lett. 113, 155003 (2014).

[18] M. E. Savage, K. R. LeChien, M. R. Lopez, B. S. Stoltzfus, W. A. Stygar, D. S. Artery, J. A. Lott, and P. A. Corcoran, Status of the $\mathrm{Z}$ pulsed power driver, in Proceedings of the 18th International Pulsed Power Conference, Chicago, Illinois (IEEE, Illinois, 2011), pp. 983-990, https://doi .org/10.1109/PPC.2011.6191629.

[19] D. V. Rose, D. R. Welch, E. A. Madrid, C. L. Miller, R. E. Clark, W. A. Stygar, M. E. Savage, G. A. Rochau, J. E. Bailey, T. J. Nash, M. E. Sceiford, K. W. Struve, P. A. Corcoran, and B. A. Whitney, Three-dimensional electromagnetic model of the pulsed-power Z-pinch accelerator, Phys. Rev. ST Accel. Beams 13, 010402 (2010).

[20] M. R. Gomez et al., Demonstration of thermonuclear conditions in magnetized liner inertial fusion experiments, Phys. Plasmas 22, 056306 (2015).

[21] S. B. Hansen et al., Diagnosing magnetized liner inertial fusion experiments on Z, Phys. Plasmas 22, 056313 (2015).

[22] P. F. Schmit et al., Understanding Fuel Magnetization and Mix Using Secondary Nuclear Reactions in MagnetoInertial Fusion, Phys. Rev. Lett. 113, 155004 (2014).

[23] P. F. Knapp et al., Effects of magnetization on fusion product trapping and secondary neutron spectra, Phys. Plasmas 22, 056312 (2015).

[24] K. D. Hahn et al., Fusion-neutron measurements for magnetized liner inertial fusion experiments on the $\mathrm{Z}$ accelerator, J. Phys. Conf. Ser. 717, 012020 (2016).

[25] D. H. Barnak, J. R. Davies, R. Betti, M. J. Bonino, E. M. Campbell, V. Yu. Glebov, D. R. Harding, J. P. Knauer, S. P. Regan, A. B. Sefkow, A. J. Harvey-Thompson, K. J. Peterson, D. B. Sinars, S. A. Slutz, M. R. Weis, and P.-Y. Chang, Laser-driven magnetized liner inertial fusion on OMEGA, Phys. Plasmas 24, 056310 (2017).

[26] J. R. Davies, D. H. Barnak, R. Betti, E. M. Campbell, P.-Y. Chang, A. B. Sefkow, K. J. Peterson, D. B. Sinars, and M. R. Weis, Laser-driven magnetized liner inertial fusion, Phys. Plasmas 24, 062701 (2017).

[27] A. J. Harvey-Thompson et al., Constraining preheat energy deposition in MagLIF experiments with multi-frame shadowgraphy, Phys. Plasmas 26, 032707 (2019).

[28] S. A. Slutz et al., Enhancing performance of magnetized liner inertial fusion at the $\mathrm{Z}$ facility, Phys. Plasmas 25, 112706 (2018).

[29] P. F. Knapp et al., Origins and effects of mix on magnetized liner inertial fusion target performance, Phys. Plasmas 26, 012704 (2019).

[30] M. R. Gomez et al., Assessing stagnation conditions and identifying trends in magnetized liner inertial fusion, in IEEE Transactions on Plasma Science (IEEE, New York, 2019), Vol. 47, pp. 2081-2101, https://doi.org/10.1109/TPS .2019 .2893517$.

[31] A. J. Harvey-Thompson et al., Diagnosing and mitigating laser preheat induced mix in MagLIF, Phys. Plasmas 25, 112705 (2018).

[32] Derek Lamppa (to be published).

[33] M. R. Gomez, R. M. Gilgenbach, M. E. Cuneo, C. A. Jennings, R. D. McBride, E. M. Waisman, B. T. Hutsel, W. A. Stygar, D. V. Rose, and Y. Maron, Experimental study of current loss and plasma formation in the $\mathrm{Z}$ machine posthole convolute, Phys. Rev. Accel. Beams 20, 010401 (2017).

[34] C. A. Hall, Isentropic compression experiments on the Sandia Z accelerator, Phys. Plasmas 7, 2069 (2000).

[35] C. L. Ruiz, G. W. Cooper, S. A. Slutz, J. E. Bailey, G. A. Chandler, T. J. Nash, T. A. Mehlhorn, R. J. Leeper, D. Fehl, A. J. Nelson, J. Franklin, and L. Ziegler, Production of Thermonuclear Neutrons from Deuterium-Filled Capsule Implosions Driven by Z-Pinch Dynamic Hohlraums, Phys. Rev. Lett. 93, 015001 (2004). 
[36] C. L. Ruiz, D. L. Fehl, G. A. Chandler, G. Cooper, B. Jones, J. D. Styron, and J. Torres, Multichannel, triaxial, neutron time-of-flight diagnostic for experiments at the Z Facility, Phys. Rev. Accel. Beams 23, 020401 (2020).

[37] R. D. McBride and S. A. Slutz, A semi-analytic model of magnetized liner inertial fusion, Phys. Plasmas 22, 052708 (2015).

[38] S. I. Braginskii, Transport processes in a plasma, in Reviews of Plasma Physics (Consultants Bureau, New York, 1965), Vol. 1, p. 205, https://static.ias.edu/pitp/2016/sites/pitp/files/ braginskii_1965-1.pdf.

[39] M. G. Haines, Heat flux effects in Ohm's law, Plasma Phys. Controlled Fusion 28, 1705 (1986).

[40] D. J. Ampleford et al., Improved morphology and reproducibility of magnetized liner inertial fusion experiments (to be published).

[41] D. R. Welch, D. V. Rose, C. Thoma, R. E. Clark, C. B. Mostrom, W. A. Stygar, and R. J. Leeper, Kinetic simulation of thermonuclear-neutron production by a $10^{7}$-A deuterium Z pinch, Phys. Plasmas 17, 072702 (2010).

[42] C. A. Coverdale, C. Deeney, A. L. Velikovich, J. Davis, R. W. Clark, Y. K. Chong, J. Chittenden, S. Chantrenne, C. L. Ruiz, G. W. Cooper, A. J. Nelson, J. Franklin, P. D. LePell, J.P. Apruzese, J. Levine, and J. Banister, Deuterium gas-puff Z-pinch implosions on the Z accelerator, Phys. Plasmas 14, 056309 (2007).

[43] P. F. Schmit and D. E. Ruiz, A conservative approach to scaling magneto-inertial fusion concepts to larger pulsedpower drivers, Phys. Plasmas 27, 062707 (2020).

[44] R. E. Olson et al., First Liquid Layer Inertial Confinement Fusion Implosions at the National Ignition Facility, Phys. Rev. Lett. 117, 245001 (2016).

[45] S. P. Regan et al., Demonstration of Fuel Hot-Spot Pressure in Excess of 50 Gbar for Direct-Drive, Layered DeuteriumTritium Implosions on OMEGA, Phys. Rev. Lett. 117, 025001 (2016). 